\title{
Improving patient engagement in self-measured blood pressure monitoring using a mobile health technology
}

\author{
Alan L. Kaplan ${ }^{*}$, Erica R. Cohen² and Eyal Zimlichman ${ }^{3}$
}

\begin{abstract}
Objective: To understand usage patterns and clinical efficacy of Hello Heart, an mHealth technology application designed to facilitate patient engagement in managing hypertension.

Methods: In this single-arm observational study, all subjects with $\geq 2$ blood pressure (BP) recordings were included. The cohort was divided into subgroups by weeks passed since download that patients were still recording measurements. Changes in BP were compared between subgroups.
\end{abstract}

Results: Of 5115 eligible subjects, 3803 (74\%) recorded BP for $\geq 2$ weeks. In the 4-week subgroup, 23\% achieved BP reduction of $\geq 10 \mathrm{mmHg}$ versus $24 \%$ in the 22 -week subgroup ( $<0.001$ ). Among 783 subjects reporting baseline hypertension $57 \%$ of the 4 -week and $69 \%$ of the 22 -week subgroups achieved BP normalization (all $p<0.001$ ).

Conclusions: We show significant decrease in BP with improved metrics over time. Higher engagement was associated with greater BP reduction and engagement was higher among those with greater clinical need of BP control.

Practice implications: Hello Heart represents an operational mHealth technology to improve patient engagement and clinical outcomes.

Keywords: mHealth, Patient engagement, Blood pressure, Hypertension

\section{Introduction}

Patient advocates, policy-makers and healthcare leaders champion an increased involvement of patients into the changing healthcare landscape [1]. Optimal execution strategies, however, remain elusive. Patient engagement is a concept that combines patients' knowledge, skills, and motivation with interventions designed to promote positive patient behaviors [2]. Patient engagement has gained wide popularity as a strategy to achieve the elusive "triple aim" of improved outcomes, better care, and lower costs [2]. The Institute of Medicine has defined patient engagement as a fundamental precursor to high-quality care, lower costs, and better health [1]. The evidence supporting the use of patient engagement techniques to

\footnotetext{
*Correspondence: alkaplan@mednet.ucla.edu

1 Department of Urology, David Geffen School of Medicine at UCLA, 10833 Le Conte Ave, Box 951738, Los Angeles, CA 90095-1738, USA Full list of author information is available at the end of the article
}

improve satisfaction and outcomes is robust [3]. Likewise, some evidence suggest that patients more involved in their care incur fewer costs overall [4]. The Centers for Medicare and Medicaid Services' (CMS) electronic health record (EHR) incentive program ["Meaningful Use"] promotes the use of health information technology to encourage patients to better understand and participate in their care [5].

Mobile health technology (mHealth) represents an important mechanism by which patients can play a larger role in managing their chronic conditions. mHealth is associated with enhanced communication between patient and provider and may improve self-management behaviors $[6,7]$. There has been immense enthusiasm surrounding mHealth over the past decade, particularly with respect to supporting self-management of chronic conditions [8]. Yet, optimal employment strategies and disease states in which mHealth may be most impactful are unknown. 
Hypertension is a prevalent chronic condition that may represent an ideal target for $m$ Health patient engagement interventions. Uncontrolled hypertension may result in end organ damage and confers increasing risk of neurovascular and cardiovascular events over time [9]. Effective blood pressure control mitigates these risks but requires diligent patient compliance and involvement in management. mHealth engagement strategies may help support patients in compliance and self-managing their treatment $[10,11]$.

In this single-arm pilot feasibility study, we sought to test usage patterns and efficacy of Hello Heart in its early launch. We hypothesized that usage patterns would be concordant with other mHealth applications, that improved engagement would yield modest BP reduction in the short term, and that longer duration of application usage would produce greater decreases in BP.

\section{Methods}

All patient-specific data were de-identified and securely encrypted. Hello Heart is Health Insurance Portability and Accountability Act (HIPPA)-compliant and anonymous data sharing is acknowledged through a priori user agreement. The institutional review board of the Chaim Sheba Medical Center at Tel HaShomer approved this research study (IRB \#2596-15-SMC).

\section{Technology design}

Hello Heart is a publicly available mHealth technology launched in April, 2015. The application provides a mobile platform through which patients can record and track self-measured BP recordings over time (Fig. 1a). Other features include periodic reminders to measure BP, interactive educational modules to improve use knowledge base, and connectivity for wireless BP measurement devices (Fig. 1b). Hello Heart was designed to maximize user engagement by incorporating mHealth best practices: ease of use, straightforward comprehension, and clarity. Screens, features and user interface were optimized toward maximal patient engagement.

The application utilizes gamification methodologies to drive user engagement. Examples include an immediate personalized feedback and reward system to maximize user interaction (Fig. 1b). Immediately following each $\mathrm{BP}$ recording event, users encounter screen displays that employ enthusiastic animation and positive reinforcement language. Personalized explanations regarding the stages of hypertension and translation into cardiovascular risk are provided for relevance and to augment user experience. The application organizes medical data in a centralized mobile platform, allows users to import that data from wireless devices (BP cuffs and wireless scales), and remotely connect Hello Heart to their physician's EHR to automatically populate laboratory and pharmacologic data. Users receive weekly BP progress reports demonstrating changes over time, thereby establishing salient healthy tracking habits, medication adherence, and weight control. Additional platforms for pharmacologic, radiologic, and laboratory input are available as well.

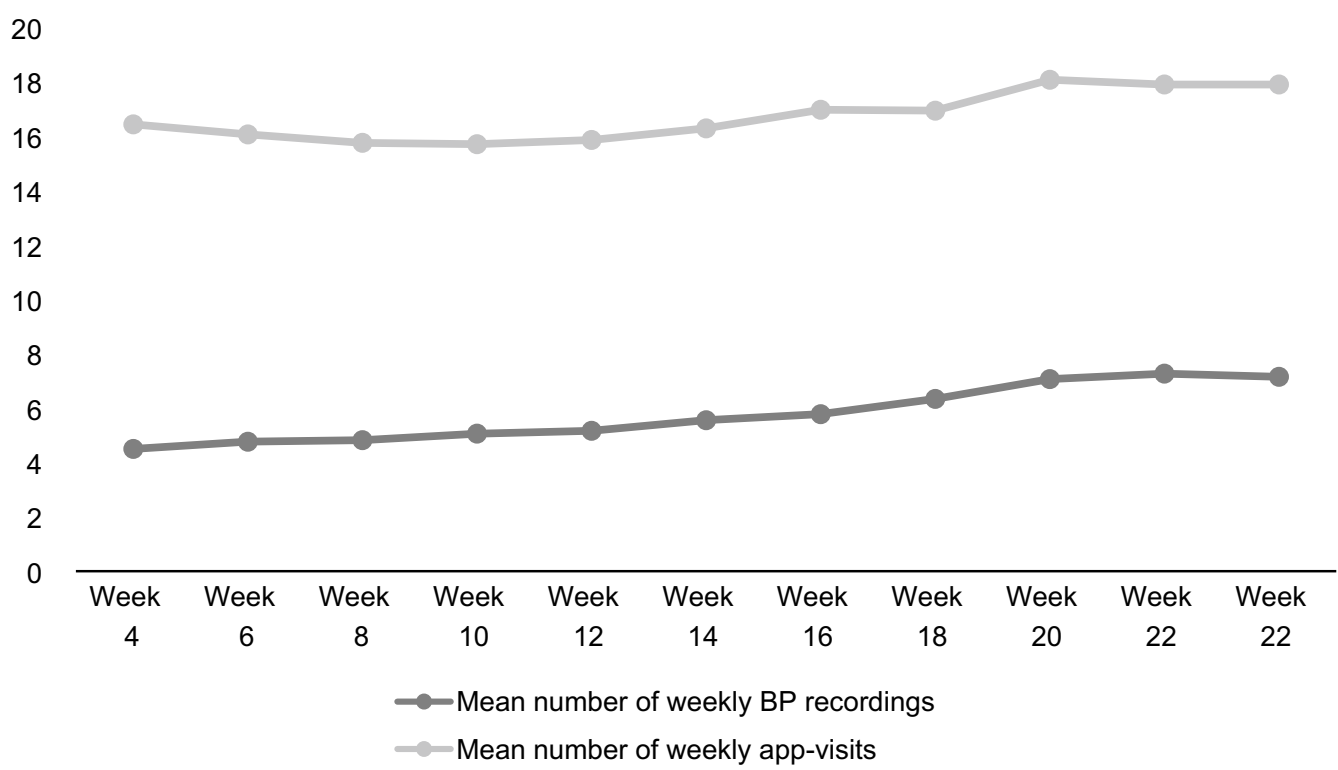

Fig. 1 Weekly blood pressure recording events versus weekly app-visits 


\section{Study cohort}

Subjects in this single-arm retrospective observational study self-enrolled online via the "Appstore" or were referred to use the application by a treating clinician for proposes of BP self monitoring. As part of the application's terms of service subjects consented to data analysis. Only subjects that recorded $\geq 2 \mathrm{BP}$ measurements $(\mathrm{n}=5115)$ were included in the study as a minimum two-point trend was necessary for effect analysis. Demographic data were self-reported when subjects initially downloaded the application and included age, gender, geographic location, comorbid conditions, and medications.

The cohort was divided into subgroups based on the number of weeks passed since application download that subjects were still recording measurements: $2,4,6$, $8,10,12,14,16,18,20,22$ weeks respectively. Subjects recording BP for less than 4 weeks were considered "low engagement", 4-8 weeks "medium engagement," and longer than 8 weeks "high engagement." Subjects persistently engaged over 22 weeks are part of the initial cohort of users from application launch in April 2015 and data collection persists.

\section{Outcomes}

The primary study objective was to understand and describe usage patterns (engagement) of the application by enrolled patients over time. For all subgroups $[2,4,6$, $8,10,12,14,16,18,20,22$ weeks passed since download, respectively], we tracked the number of weekly $\mathrm{BP}$ measurements recorded by the subject in the application. We hypothesized that there would be a precipitous "dropoff" of active users in the first 2 weeks since download, consistent with existing mHealth literature [12]. This descriptive analysis is likewise hypothesis generating in conveying demographic usage and effect trends. This data can be used to iteratively improve mHealth applications by tailoring patient engagement interventions to the end user.

Our secondary outcome assessed was the effect of usage patterns on BP. Namely, to test whether an increasing level of engagement would increase the probability of decreasing BP. A clinically significant decrease in BP was defined as a reduction of $10 \mathrm{mmHg}$ or more from beginning to end of the study period. We hypothesized that regular users would experience a modest reduction in $\mathrm{BP}$ over time. Further, we postulated that usage of the application for a longer duration (increasing level of engagement) would yield greater reductions in BP.

\section{Statistical analyses}

We used logistic regression analysis to determine efficacy by engagement level among the entire cohort. We sought to model a categorical response ("decreased", "did not decrease") given a subject's engagement level as a categorical predictor ("high", "medium", "low"). The model was then fit to those with hypertension at study outset as well. A third model was fit to test efficacy by gender, number of medications, and engagement level. We used $X^{2}$ to test independency between $10 \mathrm{mmHg}$ BP outcome (decrease, stable, increase) and engagement level. Lastly, to assess efficacy by BP subgroup (BP < 120, BP 120-140, $\mathrm{BP}>140$ ) we used the Cochran-Mantel-Haenszel test. Fitting a logistic regression model provides maximum likelihood estimates for probabilities of lowering BP by engagement category. Likewise, we can further determine whether engagement effect increases or decreases those probabilities.

\section{Results}

\section{Demographics}

We identified 5115 subjects that met inclusion criteria. Baseline demographic characteristics of the study cohort are listed in Table 1. Average age in the overall cohort was 49 years versus 59 in the 22-week subgroup. Sixty-four percent of subjects identified as male and represented wide geographic variation. Mean and systolic BP for the cohort at baseline were 130 and $128 \mathrm{mmHg}$, respectively. Sixteen percent reported depression or anxiety; $14 \%$ denied comorbid condition.

\section{Engagement}

At two weeks following application download, 3803 (74\%) were still recording BP, 2278 (45\%) at 4 weeks, 1098 $(21 \%)$ at 8 weeks, $305(6 \%)$ at 16 weeks and $99(1.9 \%)$ at 22 weeks. Mean number of weekly measurements in the 4- and 14-week subgroups was 6 and 8, respectively. Mean app visits were 15 per week across subgroups. The ratio of app-visits to BP recording was 3:1 (Fig. 2). Thirty four percent of subjects added the reminder feature of the application, while 5\% connected wireless blood pressure recording devices. Fifty-seven percent in the 4-week subgroup added a medication list compared to $72 \%$ in the 20 -week subgroup. Likewise, $74 \%$ in the 4 -week subgroup accessed their weekly BP report $\geq 3$ times versus $84 \%$ in the 12 -week and $98 \%$ in the 22 -week subgroups. The relationship between usage of specific application features and the percentage of users achieving clinically significant BP reduction is depicted in Fig. 3.

\section{Efficacy}

Among subjects in the 6-week subgroup, 63\% had stable $\mathrm{BP}$-defined as no change of $\geq 10 \mathrm{mmHg}$ since application download. Among those in the 4-week subgroup, $23 \%$ achieved a reduction of $\geq 10 \mathrm{mmHg}$ in mean systolic BP compared to $24 \%$ in the 22-week subgroup 
Table 1 Baseline cohort demographics

\begin{tabular}{|c|c|}
\hline Eligible cohort & 5115 \\
\hline Gender & $1841(36 \%)$ \\
\hline Male & $3273(64 \%)$ \\
\hline \multicolumn{2}{|l|}{ Female } \\
\hline Age & 49 (SD 13.97) \\
\hline$<30$ years old & $9 \%$ \\
\hline 31-40 years old & $16 \%$ \\
\hline $41-50$ years old & $29 \%$ \\
\hline $51-60$ years old & $21 \%$ \\
\hline$>60$ years old & $25 \%$ \\
\hline Mean systolic blood pressure & $130 \mathrm{mmHg}$ (SD 17.78) \\
\hline \multicolumn{2}{|l|}{ Medical condition } \\
\hline $\begin{array}{l}\text { Hypertension and prehypertension } \\
(>120 \mathrm{mmHg})\end{array}$ & 4165 (81\%) \\
\hline Prehypertension & 3179 (62\%) \\
\hline Hypertension stage 1 & $799(16 \%)$ \\
\hline Hypertension stage 2 & $187(4 \%)$ \\
\hline Hypercholesterolemia & $1273(25 \%)$ \\
\hline Depression and anxiety & $797(16 \%)$ \\
\hline Diabetes mellitus & $458(9 \%)$ \\
\hline $\begin{array}{l}\text { Cardiovascular disease or chronic kidney } \\
\text { disease }\end{array}$ & $365(7 \%)$ \\
\hline Chronic kidney disease & $114(2 \%)$ \\
\hline Asthma & $21(0.41 \%)$ \\
\hline Sleep apnea & $13(0.25 \%)$ \\
\hline Fibromyalgia & $12(0.23 \%)$ \\
\hline Arthritis & $10(0.2 \%)$ \\
\hline Hypothyroidism & $10(0.2 \%)$ \\
\hline Hypothyroid & $9(0.18 \%)$ \\
\hline $\mathrm{ADHD}$ & $8(0.16 \%)$ \\
\hline Allergies & $8(0.16 \%)$ \\
\hline IBS & $7(0.14 \%)$ \\
\hline Low blood pressure & $7(0.14 \%)$ \\
\hline Migraines & $7(0.14 \%)$ \\
\hline COPD & $6(0.12 \%)$ \\
\hline Lupus & $6(0.12 \%)$ \\
\hline Thyroid & $6(0.12 \%)$ \\
\hline Gout & $5(0.1 \%)$ \\
\hline I don't have a medical condition & $675(13 \%)$ \\
\hline
\end{tabular}

$(\mathrm{p}<0.001)$ (Table 2). Users in the medium and high engagement group were more likely to achieve BP reduction than those in the low engagement group, $22 \%$ versus $10 \%(\mathrm{p}<0.0001)$. Among the $783(15 \%)$ subjects reporting stage II hypertension at baseline (systolic BP $\geq 140$ ), $57 \%$ of the 4 -week, $65 \%$ of the 16 -week, and $69 \%$ of the 22 -week subgroups normalized BP to $<140$ (all p $<0.001$ ) (Fig. 4). Use of reminder feature, gender, and medication number did not affect metric outcomes (Table 3). Subjects in the high engagement group were more likely to have hypertension or pre-hypertension, cardiovascular or chronic kidney disease, and hypercholesterolemia than those in the low and medium engagement groups $(\mathrm{p}<0.0001)$ (Table 4).

Fitting the logistic regression model for BP decrease rate by engagement group yielded significant and rising effect. Coefficient estimates reflect an increased likelihood of decreasing $\mathrm{BP}>10 \mathrm{mmHg}$ on transition from low- to medium-engagement of 1.35 -namely $35 \%$ increased likelihood. Transition from medium- to highengagement yielded a coefficient estimate of 1.42 (all $\mathrm{p}<0.001)$. A similar model was fit for baseline hypertensive users yielding similar results: coefficient estimate 3.43 from low- to medium-engagement and 4.60 from medium- to high-engagement (all p $<0.001$ ). Finally, using the Cochran-Mantel-Haenszel test to assess efficacy by BP subgroup there was significant dependency between engagement level and BP effect $\left[M^{2}=16.97\right.$ $(4 d f), \mathrm{p}=0.002$ ] (Table 5).

\section{Discussion and conclusions Discussion}

The ever-changing healthcare landscape relies on progressive improvements in the value of care delivered. Value in healthcare is defined as quality of care per unit cost [13]. Patient engagement strategies necessarily improve the quality of care and may mitigate costs as well $[3,4]$. mHealth offers novel platforms by which patients can interact with the healthcare system and take ownership of their care [7]. Hello Heart is an innovative mHealth application that supports patient engagement in self-measured BP monitoring. In this pilot feasibility study, we tested usage patterns and efficacy in the early stages after application launch.

Our study has several important findings. First, users experienced a statistically and clinically significant $\mathrm{BP}$ reduction in a short period of time. In the subgroup that used the application for $<4$ weeks, $10 \%$ of subjects had a drop in systolic BP of at least $10 \mathrm{mmHg}$ ( $p<0.001$ ). After 8 weeks, $22 \%$ of subjects had realized that BP reduction ( $<<0.001$ ) versus $24 \%$ after 22 weeks. While we recognize systolic BP is subject to fluctuation from measurement to measurement, over half the users with hypertension experienced a reduction to mean systolic BP $<140 \mathrm{mmHg}$ after 4 weeks, suggesting a clinically significant effect. To understand "usual" changes in BP over time, Clark et al. performed a systematic review and meta-analysis using Cochrane data to study the control arms of 58 randomized controlled trials (RCT) [14]. In studying 6897 subjects, the authors found that mean change in BP in those randomized to control arms (i.e. usual care or non-intervention) was a decrease of $3.9 \mathrm{mmHg}(95 \% \mathrm{CI}-5.5$ to -2.4$)$. Although subjects in 


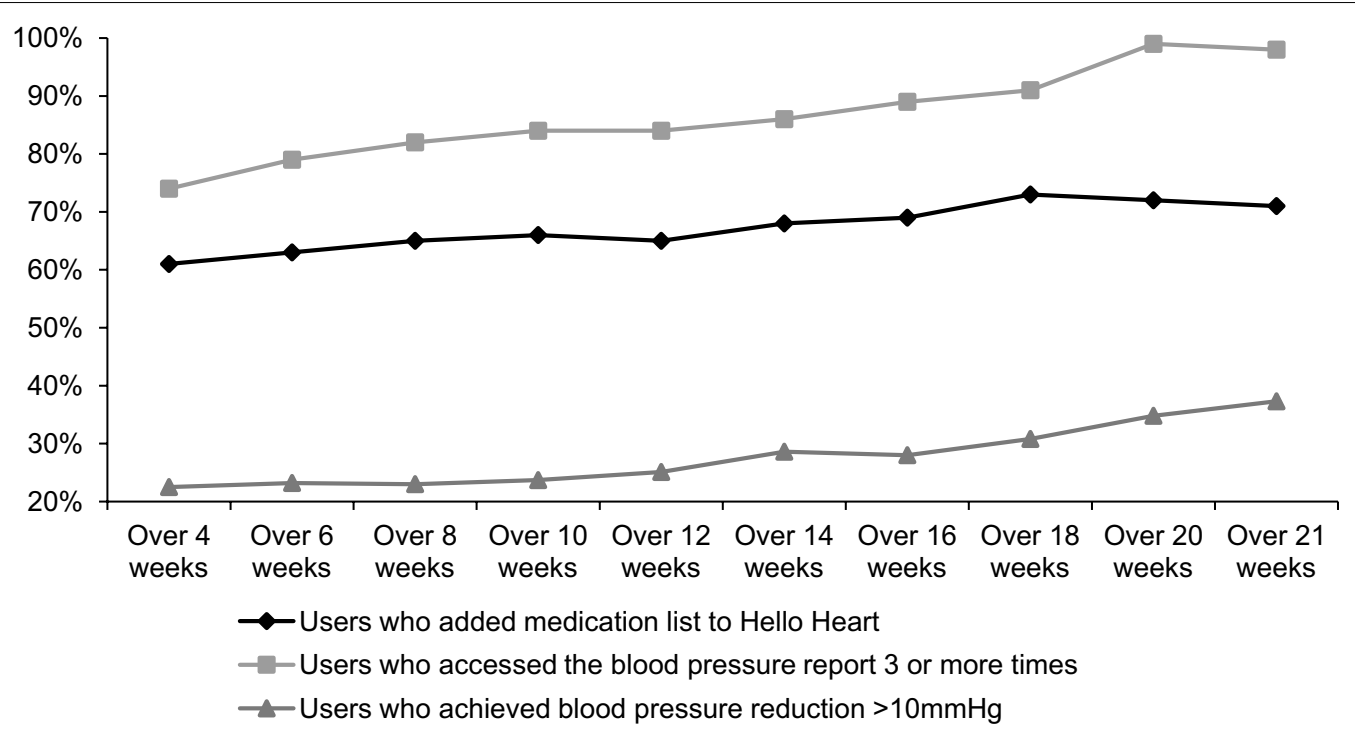

Fig. 2 Correlation between use of specific application features and blood pressure reduction

\section{$\%$ of users}

$80 \%$

$60 \%$

$40 \%$
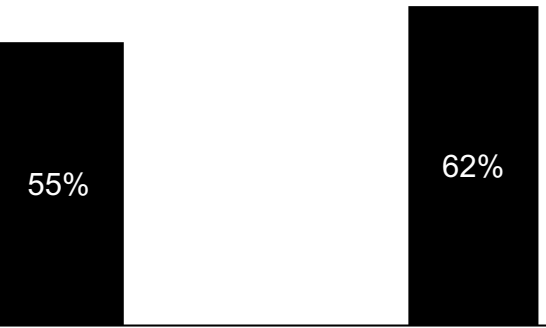

$0 \%$

*Lowered systolic blood pressure to $<140 \mathrm{mmHg}(p<0.0001)$

Fig. 3 Demonstration of blood pressure normalization by engagement level

Table 2 Blood pressure reduction by duration of application usage

\begin{tabular}{|c|c|c|c|}
\hline Subgroup & Number of patients in subgroup & $\%$ of users achieving blood pressure reduction from baseline ${ }^{a}$ & $\mathrm{p}$ value \\
\hline 4 weeks & 2278 & 22 & $<0.0001$ \\
\hline 6 weeks & 1539 & 23 & $<0.0001$ \\
\hline 8 weeks & 1098 & 22 & $<0.0001$ \\
\hline 10 weeks & 783 & 23 & $<0.001$ \\
\hline 12 weeks & 592 & 24 & $<0.001$ \\
\hline 14 weeks & 431 & 25 & $<0.0001$ \\
\hline 16 weeks & 305 & 25 & $<0.0001$ \\
\hline 18 weeks & 208 & 23 & $<0.0001$ \\
\hline 20 weeks & 142 & 25 & $<0.0001$ \\
\hline 22 weeks & 99 & 24 & $<0.001$ \\
\hline
\end{tabular}

\footnotetext{
${ }^{a}$ Defined as reduction in mean systolic blood pressure $\geq 10 \mathrm{mmHg}$
} 


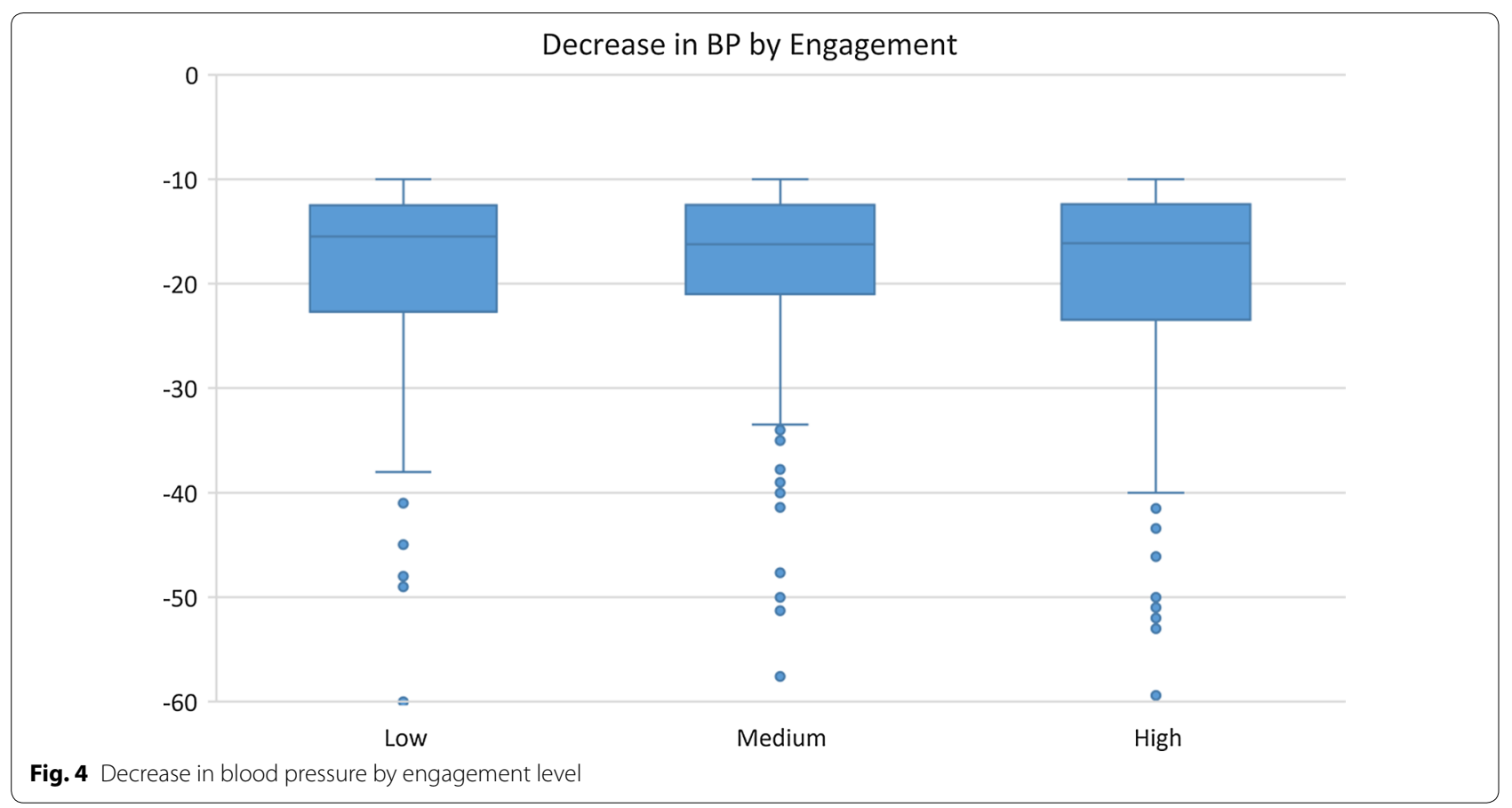

Table 3 Logistic regression model output for efficacy demonstration

\begin{tabular}{lll}
\hline Variable & Coefficient estimate (SD) & p value \\
\hline Low engagement & $1.477(0.21)$ & $<0.001$ \\
Medium engagement & $1.267(0.21)$ & $<0.001$ \\
High engagement & $1.118(0.23)$ & $<0.001$ \\
Age & $0.00071(0.0045)$ & 0.875 \\
Gender & $0.186(0.114)$ & 0.103 \\
Total medication \# & $0.000074(0.0042)$ & 0.986 \\
\hline
\end{tabular}

our cohort were not exposed to the rigorous control of an RCT and confounding effects-including Hawthorne effect from RCT enrollment-may have been present, the BP reductions achieved using Hello Heart were nonetheless striking. Several prospective studies have demonstrated increasing risk of cardiovascular disease, cerebrovascular events, and renal deterioration with progressively high blood pressure [15-17].

Second, subjects achieved more precipitous BP reduction with prolonged usage of the application. mHealth represents a powerful aspect of a larger effort to shift some of the burden of care away from the traditional medical establishment toward individual patients.

Table 4 Cohort demographics by level of engagement

\begin{tabular}{|c|c|c|c|c|}
\hline Variable & Low engagement & Medium engagement & High engagement & $p$ value \\
\hline Age & $46(S D 14.0)$ & 59 (SD 13.1) & 54 (SD 13.2) & - \\
\hline Gender & 135 & 123 & 116 & - \\
\hline Male & $64 \%$ & $64 \%$ & $63 \%$ & - \\
\hline Female & $36 \%$ & $36 \%$ & $37 \%$ & - \\
\hline Number of medications & $3(s d 2.9)$ & $4(\mathrm{sd} 3.8)$ & $5(\mathrm{sd} 4.3)$ & - \\
\hline \multicolumn{5}{|l|}{ Comorbidities } \\
\hline Hypertension or pre-hypertension & $2127(75 \%)$ & $1036(88 \%)$ & $1002(91 \%)$ & $<0.0001$ \\
\hline Hypercholesterolemia & $662(23 \%)$ & $297(25 \%)$ & $284(26 \%)$ & $<0.0001$ \\
\hline Depression and anxiety & $476(17 \%)$ & $184(16 \%)$ & $124(11 \%)$ & $<0.0001$ \\
\hline Diabetes mellitus & $259(9 \%)$ & $95(8 \%)$ & $99(9 \%)$ & $<0.0001$ \\
\hline Cardiovascular disease or chronic kidney disease & $198(7 \%)$ & $77(7 \%)$ & $98(9 \%)$ & $<0.0001$ \\
\hline None reported & $397(14 \%)$ & $136(12 \%)$ & $111(10 \%)$ & $<0.0001$ \\
\hline
\end{tabular}


Table 5 Self-reported medication use

\begin{tabular}{ll}
\hline Medication & \% Reporting medication \\
\hline ACE inhibitor & 8.63 \\
Beta blocker & 7.28 \\
Cholesterol lowering & 7.24 \\
Calcium channel blocker & 5.06 \\
Diuretic & 4.80 \\
Antiplatelet agent & 4.28 \\
ARB & 3.64 \\
Anticoagulant & 0.41 \\
Vasodilator & 0.23 \\
Digitails preparation & 0.11 \\
\hline
\end{tabular}

mHealth interventions are mediated through behavioral change theory and may improve patient engagement [18]. Widmer et al. conducted a systematic review and meta-analysis to determine whether digital health interventions impacted cardiovascular disease outcomes and risks factors [19]. Cardiovascular disease outcomes were defined as cardiovascular events, hospitalizations, and mortality. Risk factors were defined as weight, blood pressure, and hypercholesterolemia. The authors found that digital health interventions significantly reduced the number of cardiovascular disease outcomes. Additionally, they found that these interventions reduced weight and body mass index but not blood pressure [19]. In our study, we were able to show reduction in BP over a short period of time that improved the longer subjects used the application. Although some studies have been able to demonstrate decreased BP using mHealth technologies, these studies enrolled subjects with high-risk hypertensive disease and specific comorbid conditions [20, 21]. Recent Cochrane reviews found limited evidence to support these interventions [8, 22]. But given conflicting data, those authors concluded that further studies are needed to elucidate the role of mHealth in chronic conditions.

Third, among regular users, engagement was high. At 2 weeks, $74 \%$ of subjects that had begun using the application were still regularly inputting their BP measurements. Likewise, at 22-weeks, users were accessing their $\mathrm{BP}$ reports $\geq 3$ times per week. That the mean number of BP measurements recorded per week for users who continued recording blood pressure at 6 weeks was 6 compared to 8 among those who continued for at least 14 weeks, demonstrates high levels of engagement with respect to self-measured BP monitoring. In a comparative effectiveness review supported by the Agency for Healthcare Research and Quality (AHRQ), Uhlig et al. showed that self-measured BP monitoring provided a modest benefit in BP control compared with usual care. The authors found insufficient evidence to define predictors of adherence to self-measured BP monitoring [23]. In our study, the app visit to BP recording ratio was 3:1. This indicates users are engaging in other components of the application (i.e. disease-specific education, adding personal health data). This in-depth interaction, which is increasing over time, suggests users are engaging in their personal health with increasing usage of the application.

Finally, users with the highest clinical need demonstrated the highest levels of engagement. Namely, subjects in the high engagement group were more likely to suffer from hypertension or pre-hypertension, cardiovascular or chronic kidney disease, and hypercholesterolemia (all $\mathrm{p}<0.0001$ ). Not surprisingly, but importantly, the differences in engagement were most pronounced among those with, or at risk of developing, hypertension, $91 \%$ versus $75 \%(\mathrm{p}<0.0001)$. This would suggest the application is effectively capturing the users that need to be captured from a clinical standpoint.

To frame context for efficacy of mHealth applications to lower BP, we conducted a systematic review of the literature. Applying pertinent English-language search terms - health, mobile, hypertension-we identified 169 applicable studies. Thirty-two studies used mobile technology and demonstrated a decrease in BP among users with an average systolic BP reduction of $8.8 \mathrm{mmHg}$ (SD 4.2). We identified 3 studies in the literature that used mobile application platforms to engage users in BP management. Eccher et al. describe the use of a mobile logbook that uses software linking wireless BP tracking devices [24]. Outcomes assessed were user acceptability, compliance, and perceived benefits and disadvantages by both user and physician. Chen et al. used an at-home telehealth BP monitoring service to facilitate communication between patient and provider through video chat software [25]. The authors found that the telehealth service attained better BP control when compared to selfcheck alone. Primary outcome was BP control rather than reduction. Finally, Mann et al. tested an mHealth application to improve BP by tracking dietary portion control, $\mathrm{BMI}$, wireless BP monitoring, and interactive feedback loops [26]. The primary outcome measured was usability. The primary difference between Hello Heart and these studies is integration of multiple feature interfaces (i.e tracking, gamification, education, and notification alerts) versus single feature. Secondarily, demonstration of clinically meaningful BP reduction is both salient and unique.

These findings must be interpreted in the context of the study design. First, the retrospective observational design limits our ability to determine whether BP reductions were directly related to use of the application or other confounding factors. Associations identified in such a 
study do not imply causation. Nonetheless, Hello Heart is a patient engagement mHealth application designed to encourage patient involvement in chronic condition management. Although secondary confounding benefit may be at play, improved patient engagement and overall health is the ultimate objective. Second, the followup period is relatively short. We did identify increased "drop-off" with each week passed although the outcome metrics improved over time. We are currently exploring iterative modifications to the application design to mitigate "drop-off"-an issue of particular importance in those subjects who experienced clinically meaningful BP reduction. Finally, while hypertension is an important modifiable risk factor for cardiovascular disease, it accounts for a narrow segment of overall health. Future directions include wellness interventions that extend beyond the BP realm.

\section{Conclusions}

We describe usage patterns and efficacy of an mHealth application designed to improve patient engagement in self-measured BP monitoring. Improved patient engagement is associated with better health outcomes, higher patient satisfaction, and lower cost of care. We show a significant reduction in systolic BP in a short timeframe. Among regular users, patient engagement was high. Subjects that used the application for longer duration experienced greater reduction in BP from their baseline.

\section{Practice implications}

In this pilot feasibility study, Hello Heart shows promise as an accessible tool and potential mechanism by which patients and providers may improve patient engagement in the management of hypertension. The application may be of benefit to patients with or at risk for hypertension and treating clinicians. Additionally, payors and large employers may employ this type of mHealth intervention to improve overall health and wellness in a managed population. Further studies are needed to fully understand how best to adopt mHealth into clinical practice or consumer wellness programs to improve patient engagement and value in healthcare.

\section{Authors' contributions}

ALK carried out the data analyses, interpreted the data and drafted the manuscript. ERC assisted in analytic interpretation and in drafting the manuscript. EZ edited the manuscript and helped with research study design. All authors read and approved the final manuscript.

\section{Author details}

1 Department of Urology, David Geffen School of Medicine at UCLA, 10833 Le Conte Ave, Box 951738, Los Angeles, CA 90095-1738, USA. ${ }^{2}$ Department of Medicine, Cedars-Sinai Medical Center, Los Angeles, CA, USA. ${ }^{3}$ Department of Medicine, Sheba Medical Center, Ramat Gan, Israel.

\section{Acknowledgements}

Not applicable.
Competing interests statement

Dr. Alan L. Kaplan is a paid consultant for Hello Heart. Dr. Eyal Zimlichman is an advisory board member of Hello Heart and holds stock options with the company. Dr. Erica R. Cohen declares no competing interests.

\section{Availability of data and materials}

Data described in this study are proprietary in the context of further research and analytics.

\section{Consent for publication}

Not applicable.

\section{Ethics approval and consent to participate}

The institutional review board of the Chaim Sheba Medical Center at Tel HaShomer approved this research study (IRB \#2596-15-SMC). Consent to participate was obtained from all subjects.

Funding

Not applicable.

\section{Publisher's Note}

Springer Nature remains neutral with regard to jurisdictional claims in published maps and institutional affiliations.

Received: 12 April 2016 Accepted: 31 August 2017

Published online: 07 October 2017

\section{References}

1. Matter Front. Partnering with patients to drive shared decisions, better value, and care improvement: workshop proceedings. Washington, DC: The National Academies Press; 2014.

2. Health policy brief. Patient engagement. Health Aff (Millwood). February 2013

3. Hibbard JH, Greene J. What the evidence shows about patient activation: better health outcomes and care experiences. Fewer data on costs. Health Aff. 2013;32:207-14.

4. Hibbard JH, Greene J, Overton V. Patients with lower activation associated with higher costs; delivery systems should know their patients"scores'. Health Aff (Millwood). 2013;32:216-22.

5. CMS. Medicare and medicaid programs; Electronic health record incentive program-stage 2, 2012. https://www.cms.gov/Regulations-andGuidance/Legislation/EHRIncentivePrograms/Stage_2.html. Accessed 30 Aug 2015.

6. Jolles EP, Clark AM, Braam B. Getting the message across: opportunities and obstacles in effective communication in hypertension care. J Hypertens. 2013;30:1500-10.

7. Logan AG. Transforming hypertension management using mobile health technology for telemonitoring and self-care support. Can J Cardiol. 2013;29:579-85.

8. de Jongh T, Gurol-Urganci I, Vodopivec-Jamsek V, et al. Mobile phone messaging for facilitating self-management of long-term illnesses. Database Syst Rev. 2012;12:CD007459.

9. Chobanian AV, Bakris GL, Black HR, et al. Seventh report of the Joint National Committee on Prevention, Detection, Evaluation, and Treatment of High Blood Pressure. Hypertension. 2003:42:1206-52.

10. Eckel RH, Jakicic JM, Ard JD, et al. 2013 AHA/ACC guideline on lifestyle management to reduce cardiovascular risk: a report of the American College of Cardiology/American Heart Association Task Force on Practice Guidelines. Circulation. 2014;129(25 Suppl 2):S76-99.

11. Hallberg I, Ranerup A, Kjellgren K. Supporting the self-management of hypertension: patients' experiences of using a mobile phone-based system. J Hum Hypertens. 2015; doi:10.1038/jhh.2015.37.

12. Cipolla R. The app churn risk window: $60 \%$ chance users who don't come back within 7 days never return. Localytics 2014. http://info.localytics. com/blog/the-app-churn-risk-window. Accessed 17 Nov 2015.

13. Porter ME. What is value in health care? N Engl J Med. 2010;363:2477-81. 
14. Clark C, Smith L, Glynn L, et al. Changes in blood pressure in patients with hypertension receiving usual care in randomized controlled trials. Findings from a systematic review and meta-analysis. J Hypertens. 2015;33(Suppl 1):e85. doi:10.1097/01.hjh.0000467579.50864.5d.

15. MacMahon S, Peto R, Cutler J, et al. Blood pressure, stroke, and coronary heart disease, part 1: prolonged differences in blood pressure: prospective observational studies corrected for the regression dilution bias. Lancet. 1990;335:765-74.

16. Klag MJ, Whelton PK, Randall BL, et al. Blood pressure and end-stage renal disease in men. N Engl J Med. 1996;334:13-8.

17. He J, Whelton PK. Elevated systolic blood pressure and risk of cardiovascular and renal disease: an overview of evidence from observational epidemiologic studies and randomized controlled trial. Am Heart J. 1999;138:S211-9.

18. Pagoto S, Bennett GG. How behavioral science can advance digital health. Tansl Behav Med. 2013;3:271-6.

19. Widmer RJ, Collins NM, Collins CS, et al. Digital health interventions for the prevention of cardiovascular disease: a systematic review and metaanalysis. Mayo Clin Proc. 2015;90:469-80.

20. Logan AG, Mclssac WJ, Tisler A, et al. Mobile phone-based remote patient monitoring system for management of hypertension in diabetic patients. Am J Hypertens. 2007;20:942-8.
21. Logan AG, Irvine MJ, Mclssac WJ, et al. Effect of home blood pressure telemonitoring with self-care support on uncontrolled systolic hypertension in diabetics. Hypertension. 2012;60:51-7.

22. Glynn LG, Murphy AW, Smith SM, et al. Interventions used to improve control of blood pressure in patients with hypertension. Cochrane Database Syst Rev. 2010;3:182.

23. Uhlig K, Balk EM, Patel K, et al. Self-measured blood pressure monitoring: comparative effectiveness. AHRQ publication no. 12-EHC002-EF. Rockville, MD: Agency for Healthcare Research and Quality; 2012.

24. Eccher C, Botteri M, Ortolani M, et al. A mobile logbook to diagnose masked hypertension: a pilot application. Stud Health Technol Inform. 2014;205:363-7.

25. Chen MJ, Chen KY, Chiang SJ, et al. A telehealth service model for the treatment of hypertension. J Telemed Telecare. 2013;19:238-41.

26. Mann DM, Kudesia V, Reddy S, et al. Development of DASH Mobile: a mHealth lifestyle change intervention for the management of hypertension. Stud Health Technol Inform. 2013;192:973. 